\section{Rate of Pedestrian Signal Phase Actuation as a Proxy Measurement of Pedestrian Demand}

Christopher M. Day, Hiromal Premachandra, and Darcy M. Bullock

PuRdue

\begin{tabular}{llllllllll}
\hline$U$ & $N$ & $I$ & $V$ & $E$ & $R$ & $S$ & $I$ & $T$ & $Y$
\end{tabular}

Paper No. 10-0220



Does signal event da
in pedestrian activity?

Use rate of actuation of the pedestrian phase as a proxy measure of pedestrian activity

How does the rate pedestrian phase actuation

actuation after the implementation of an exclusive pedestrian phase?

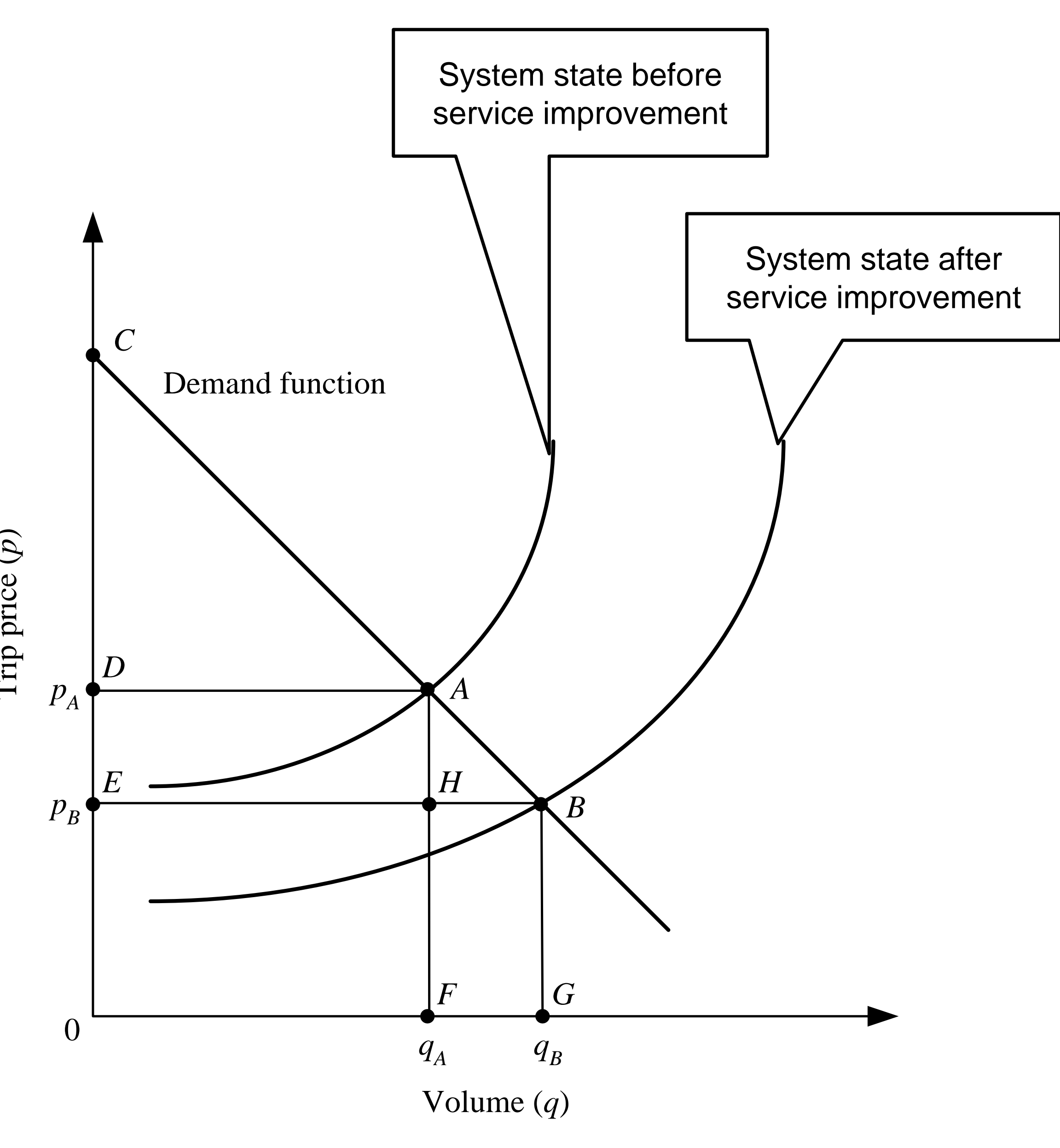

\section{Abstract}

Tor a number of ap milications higher quality data on pedestrian demand pattent heatth, and other areas. It is particularly desirable to better characterize the special events; ; and the effects of changeses in pedestrian phasing. This paper ( eedestrian count, it can be used as a surrogate to characterize how pedestrian
volumes vary due to operating conditions. The methodology could be used an

This paper documents findings from over a year of data collection at a signalized secial events, weather (temperarature and precipitatition), seasonal changes in .

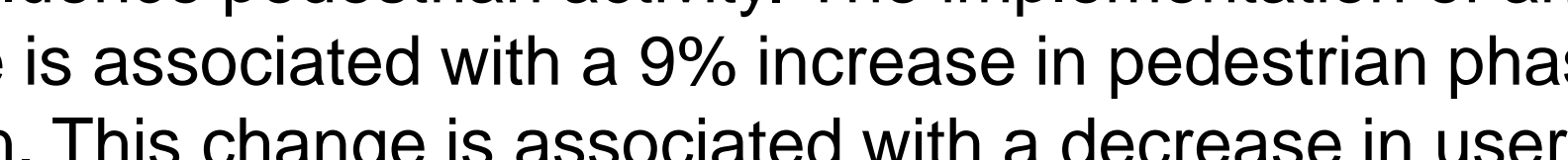

\section{Research Questions}




\section{Rate of Pedestrian Signal Phase Actuation as a Proxy Measurement of Pedestrian Demand}

Christopher M. Day, Hiromal Premachandra, and Darcy M. Bullock
PURDUE

\begin{tabular}{llllllllll}
\hline$U$ & $N$ & $I$ & $V$ & $E$ & $R$ & $S$ & $I$ & $T$ & $Y$
\end{tabular}

Paper No. 10-0220
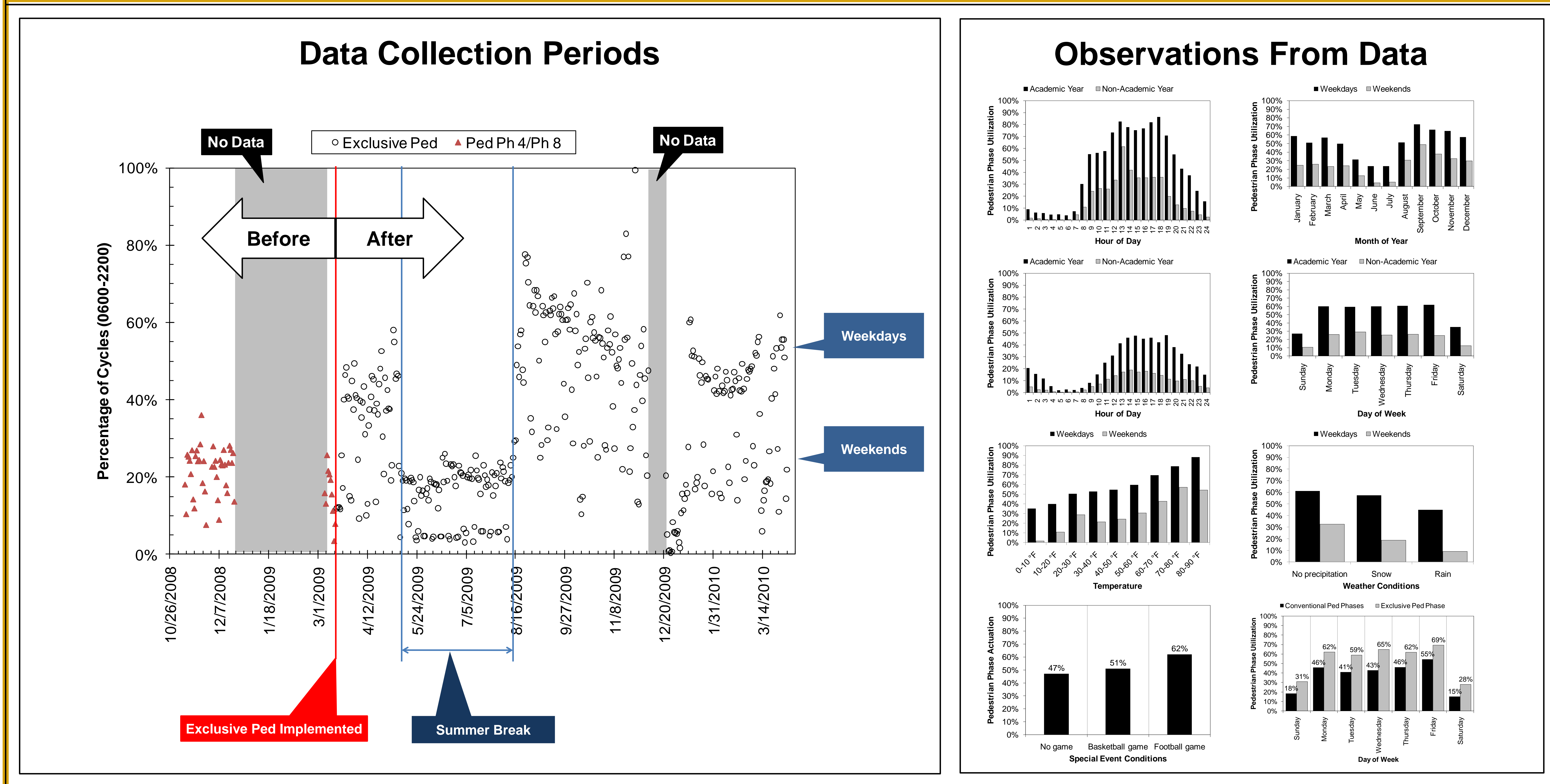

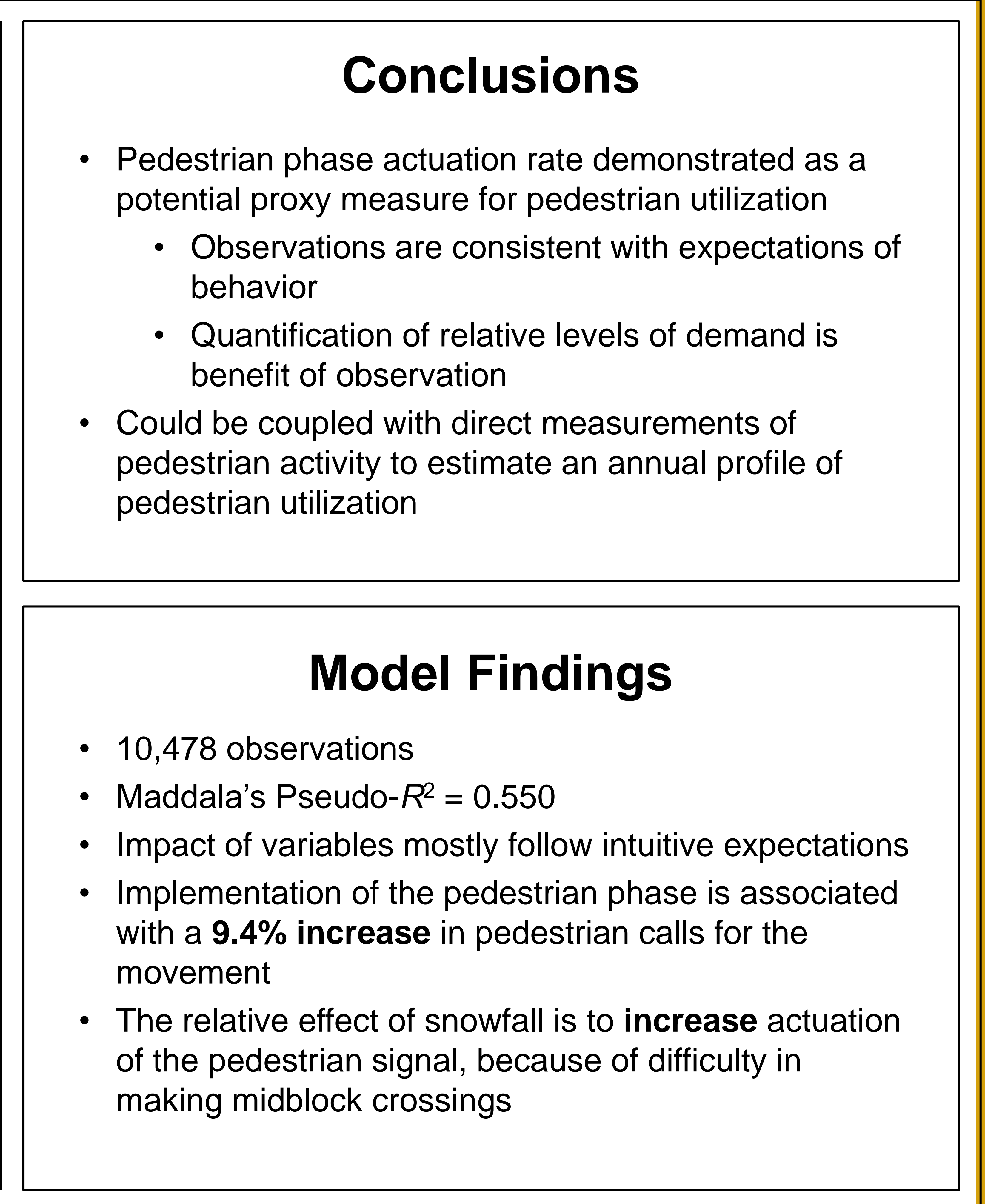

Tobit Model Specification and Variable Descriptive Statistics



